\title{
Au-delà des frontières. Les lignées de Basse-Navarre à la cour
} du roi

Ana Zabalza Seguín, Luis Erneta Altarriba

\section{Citer ce document / Cite this document :}

Zabalza Seguín Ana, Erneta Altarriba Luis. Au-delà des frontières. Les lignées de Basse-Navarre à la cour du roi. In: Nouvelle revue d'onomastique, $n^{\circ} 59,2017$. Noms de lieux, noms de personnes : la question des sources. Toponymie urbaine de Paris et de sa banlieue. Actes du XVIle colloque de la Société française d'onomastique, 2-5 décembre 2015. Volume 1. pp. 83-98;

doi : https://doi.org/10.3406/onoma.2017.1875

https://www.persee.fr/doc/onoma_0755-7752_2017_num_59_1_1875

Fichier pdf généré le 07/05/2021 


\section{Ana Zabalza Seguin - Luis Erneta Altarriba \\ Université de Navarre}

\section{Au-delà des frontières. Les lignées de Basse-Navarre à la cour du roi}

\section{Introduction. À propos des sources}

Dans les études d'onomastique réalisées dans une perspective historique, comme celle-ci, les sources sont un vrai défi pour l'imagination. L'intention du rédacteur coïncide rarement avec l'intérêt de l'historien ; cependant, plus on recule dans le temps, plus l'étude minutieuse des différentes façons de dénommer les personnes est importante, car ces dernières renferment les clés pour déterminer, par exemple, à quel groupe ethnique appartenaient ceux qui ont peuplé un lieu. Il n'est pas nécessaire de remonter le temps jusqu'à d'antiques périodes : à l'époque moderne encore, notamment au XVI ${ }^{\mathrm{e}}$ siècle, il est souvent compliqué de recouper tous les documents dont on dispose, à cause de la difficulté à vérifier, par exemple, que plusieurs documents se réfèrent en réalité à une même personne qui répond à différents noms; ou que deux sujets sont frères, alors qu'ils ont le même prénom mais des noms différents. Ces circonstances, qui peuvent être considérées comme un problème de plus pour la recherche historique, constituent d'un autre point de vue une donnée nouvelle et utile ${ }^{1}$. Comme chacun sait, avant la mise en place des États modernes il n'existait pas d'institution publique chargée d'enregistrer le nom officiel des individus. Par conséquent, il n'y a pas de nom officiel, véritable, correct, mais - comme c'est encore le cas aujourd'hui - une personne peut être connue sous différents noms en fonction de l'endroit où elle se trouve. D'ailleurs, avant la naissance de l'État moderne, un individu n'utilisait pas son propre nom, ce sont les autres qui le faisaient pour l'appeler ${ }^{2}$.

S'engager dans une recherche onomastique portant sur le début de l'époque moderne ou reconstruire des ascendances qui ont leurs racines à la fin du Moyen Âge est aussi complexe que passionnant. Dans l'exemple que nous présentons ici, nous avons essayé de reconstruire la trajectoire d'une lignée originaire de Basse-Navarre, territoire qui, jusqu'en 1530 environ, faisait partie du royaume de Navarre mais qui fut abandonné quelques années après la conquête castillane et, après diverses péripéties, devint territoire français. Pendant des siècles, beaucoup de Bas-Navarrais, qui furent attirés par l'existence de terres disponibles en Navarre, émigrèrent temporairement ou définitivement vers les terres du Sud ; témoignage de cette émigration, restent aujourd'hui encore leurs noms, souvent le

\footnotetext{
${ }^{1}$ Wrigley 1973 , p. 5.

${ }^{2}$ Ce travail s'inscrit dans le cadre du programme de recherche du Ministerio de Ciencia e Innovación DER201239334, « La integración de territorios en nuevas entidades políticas y sus consecuencias en las instituciones administrativas », dirigé par Mercedes GALÁN LORDA (Universidad de Navarra).
}

Ana Zabalza Seguin et Luis Erneta Altarriba, « Au-delà des frontières. Les lignées de Basse-Navarre à la cour du roi », Nouvelle Revue d'Onomastique ${ }^{\circ}$ 59, 2017, p. 83-98. 
nom de leur lieu d'origine, parmi lesquels Sola ou Armendáriz sont les plus communs actuellement en Navarre.

Le royaume de Navarre possède un extraordinaire ensemble de documents médiévaux, conservés à l'Archivo Real y General de Navarre. La quasi-totalité en a été numérisée pour garantir leur conservation; il existe également de bons instruments de recherche. Cet ensemble présente un trait très significatif : la plus grande partie en est constituée par la comptabilité du royaume ${ }^{3}$. Paiements, reçus, dons, exemptions ont été collectés avec une extraordinaire minutie. Cependant, les actes de gouvernement sont rares : en 1234, la dynastie royale autochtone s'éteignit et à partir de cette date, la Navarre fut régie par les rois français, qui se montrèrent toujours plus intéressés par ce qui se passait sur leurs territoires d'origine que sur ce petit territoire, pauvre et peu peuplé. La situation ne changea pas avant le dernier quart du XIV ${ }^{\mathrm{e}}$ siècle, quand le roi Charles II (1349-1387), de la dynastie d'Évreux, définitivement vaincu en France, n'eut d'autre choix que de se replier sur la Navarre. Durant le règne de son fils Charles III (1387-1425), ce monarque, résigné sans doute à son sort, essaya de donner le plus de splendeur possible à sa petite cour ; pour ce faire, de nouveaux titres pompeux furent créés; deux nouveaux palais royaux, luxueux, furent construits à Olite et à Tafalla, et splendidement décorés. En fait, la cour d'Olite était une vitrine qui permettait d'échapper à l'étroitesse des horizons d'un royaume qui se trouvait sous la pression de voisins de plus en plus puissants et ambitieux : la France, la Castille et l'Aragon ${ }^{4}$.

De tout cela nous sont restés des milliers de témoignages dans la documentation de la Cámara de Comptos, l'institution chargée de la comptabilité de la couronne. Il s'agit d'une source très précieuse du point de vue onomastique aussi, car il est possible de d'établir des biographies en suivant les attestations dans des livres de comptes et de déterminer les relations entre des personnes et des familles. Dans le cas de lignées comme celle que nous avons choisie pour cette étude, et qui ont joué un rôle secondaire à la cour, l'on peut ainsi trouver plus de cent documents par individu pour la période des règnes de Charles II et de Charles III. En recoupant les informations disponibles sur les différents membres du groupe, il est possible de tirer de nouvelles conclusions et de quantifier le volume de bienfaits ou de grâces de différentes natures que la lignée reçut de la Couronne. Par ailleurs, le fait que différentes mains mettent par écrit des prénoms et noms basques, germaniques ou romans permet de suivre leurs variantes formelles, ce qui constitue également une donnée exploitable.

Le territoire qui composait la Navarre à la fin du Moyen Âge s'était constitué durant une longue période, et tous ses espaces ne présentaient pas la même cohérence. Aux deux extrémités géographiques de l'ancien royaume on trouve ainsi deux entités d'intégration relativement tardive. D'une part, au sud, la vallée de l'Èbre, conquise sur les musulmans en 1119 environ ; autour de Tudela, la ville principale, dominaient de grands espaces, aptes à l'agriculture, de type méditerranéen. À l'extrémité nord, au-delà de la barrière

\footnotetext{
${ }^{3}$ Martinena Ruiz 1997, p. 92.

${ }^{4}$ Je remercie ma collègue, Dr Raquel GARCía ARANCÓN, pour cette information.
} 


\section{Au-delà des frontières. Les lignées de Basse-Navarre à la cour du roi}

pyrénéenne, la Basse-Navarre - également connue sous le nom d'Ultrapuertos, “OutreMonts" -, présentait des caractéristiques physiques et humaines très différentes. Rattachée à la couronne de Navarre depuis 1189 , c'est une région au relief accidenté, qui présente, sur peu de kilomètres, de forts contrastes depuis les premiers sommets pyrénéens jusqu'aux vallées de faible altitude. Avec un climat humide et peu de terrains cultivables, elle était relativement surpeuplée. L'accès à la terre, le bien par excellence, était ici impossible. Comme dans d'autres régions montagneuses européennes, la recherche du difficile équilibre entre population et ressources finit par se cristalliser dans le système de l'héritier unique, qui dans la pratique empêchait l'augmentation du nombre d'unités domestiques, avec pour conséquence l'exhérédation des cadets. Depuis le rattachement à la couronne de Navarre, les habitants de Basse-Navarre ont certainement dû voir les grands espaces du Sud comme un pays de cocagne : pour les déshérités, il n'existait en effet guère de débouchés, sinon le traditionnel service militaire en tant que mercenaires.

Les dénominations toponymiques individuelles ou familiales ainsi que les descriptions et désignations de villes, châteaux ou maisons fortes, aident à identifier la pléiade de centres de pouvoir constitués par les diverses familles qui jouissent de la faveur du roi. Elles se traduisent sur le territoire par un réseau d'influences dans la compétition pour le pouvoir sur l'espace conquis ou contrôlé.

L'analyse territoriale a été effectuée, d'une part, sur la base de la documentation consultée dans les archives et, d'autre part, avec l'information cartographique élaborée par les chercheurs spécialisés. Cela a permis de contextualiser l'espace occupé par les places fortes les plus importantes de l'ancien royaume dans l'objectif de représenter dans l'espace les connexions personnelles et familiales de la Cour, ce qui fournit de riches informations non seulement sur leur position mais aussi sur leur hiérarchie et leur fonction dans le royaume.

Comme on l'a indiqué plus haut, les rois de France montrèrent en général peu d'intérêt pour les problèmes de ce royaume. Mais quand les monarques de la dynastie d'Évreux se centrèrent sur ce petit territoire, les choses changèrent : ce n'est pas un hasard si Charles III décida la construction de ses deux palais à Olite et Tafalla, précisément là où la Navarre montagneuse s'ouvre sur la vallée de l'Èbre, sur une route qui communiquait avec les royaumes voisins. En ce qui concerne la Basse-Navarre, à son détachement tardif s'ajoutait la barrière montagneuse des Pyrénées. En outre, cette société se caractérisait par un réseau très dense de lignées, parmi lesquelles un grand nombre d'exempts d'impôts, qui, dans certaines vallées, atteignaient $66 \%$. Le contrôle de cet espace divisé en camps et en factions n'a pas dû être facile. Déjà sous Charles II, mais surtout sous son fils Charles III, on discerne précisément, dans le fonds de la Cámara de Comptos, quelles ont été les voies empruntées par les rois pour obtenir la pacification et les prélèvements fiscaux dans leurs domaines du nord des Pyrénées : ils choisirent d'attirer les membres des principales lignées, en les mettant à leur service, en leur offrant des charges et en s'assurant leur fidélité grâce à toutes sortes de récompenses. Voilà l'information qui est parvenue jusqu'à nous. 


\section{La lignée Lizarazu - Santa María}

Les Lizarazu, selon tous les indices, devaient constituer une famille typique de la petite noblesse bas-navarraise. Les premières mentions dans les sources remontent au moins à 1269 et, comme cela est fréquent dans les reconstructions généalogiques, certains de ses membres sont qualifiés de fortissimus baro. Ils sont connus sous un nom qui est en réalité celui d'un de leurs palais, qui, comme tant d'autres, est exempt de taxes et a une certaine importance au niveau purement local.

Comme beaucoup de leurs voisins, poussés par la nécessité, les Lizarazu se consacrèrent au métier des armes. Pour cela, ils se firent mercenaires au service de quiconque voulant bien les engager. Les informations les concernant commencent à être plus nombreuses sous le règne de Charles II, le dernier des monarques de la dynastie d'Évreux, qui essaya par tous les moyens de faire valoir ses droits en France. Dans ce contexte, en 1369, nous rencontrons un Lizarazu du nom de Perusco signant un contrat en tant que mercenaire au service du monarque navarrais ; il dut lever le gage de son harnais avant de s'enrôler dans l'armée, ce qui donne une idée de la précarité de sa situation.

Dans l'arbre généalogique, on peut suivre les péripéties de cette lignée, dans la mesure où les sources le permettent. Nous connaissons très peu de noms de femmes ayant appartenu à cette lignée jusqu' au $\mathrm{XV}^{\mathrm{e}}$ siècle, à une exception notable que nous analyserons un peu plus loin. Comme on peut le voir, les fils aînés de la branche principale reçurent alternativement les noms de Pedro et de Sancho, qui étaient ceux de certains rois de la dynastie autochtone navarraise éteinte en 1234 - le dernier de ces souverains fut Sancho el Fuerte "Sancho le Fort". Outre le prénom, le patronyme était fréquent, notamment chez ceux qui s'appelaient Pedro : par exemple, Pedro Sánchez de Lizarazu ; mais l'on rencontre aussi la dénomination Pedro Sanz de Lizarazu, où Sanz est une variante du prénom Sancho. Nous n'avons trouvé aucun cas d'un Sancho à qui l'on aurait donné le patronyme Pérez, mais il est vrai que l'information les concernant est insuffisante. Au prénom était ajouté un nom relié par la préposition de : par exemple, Sancho de Lizarazu ou de Liçaraçu. D'autres branches secondaires de la lignée ont des pratiques analogues, bien qu'on ne perçoive pas aussi clairement la transmission grand-père/petit-fils. 


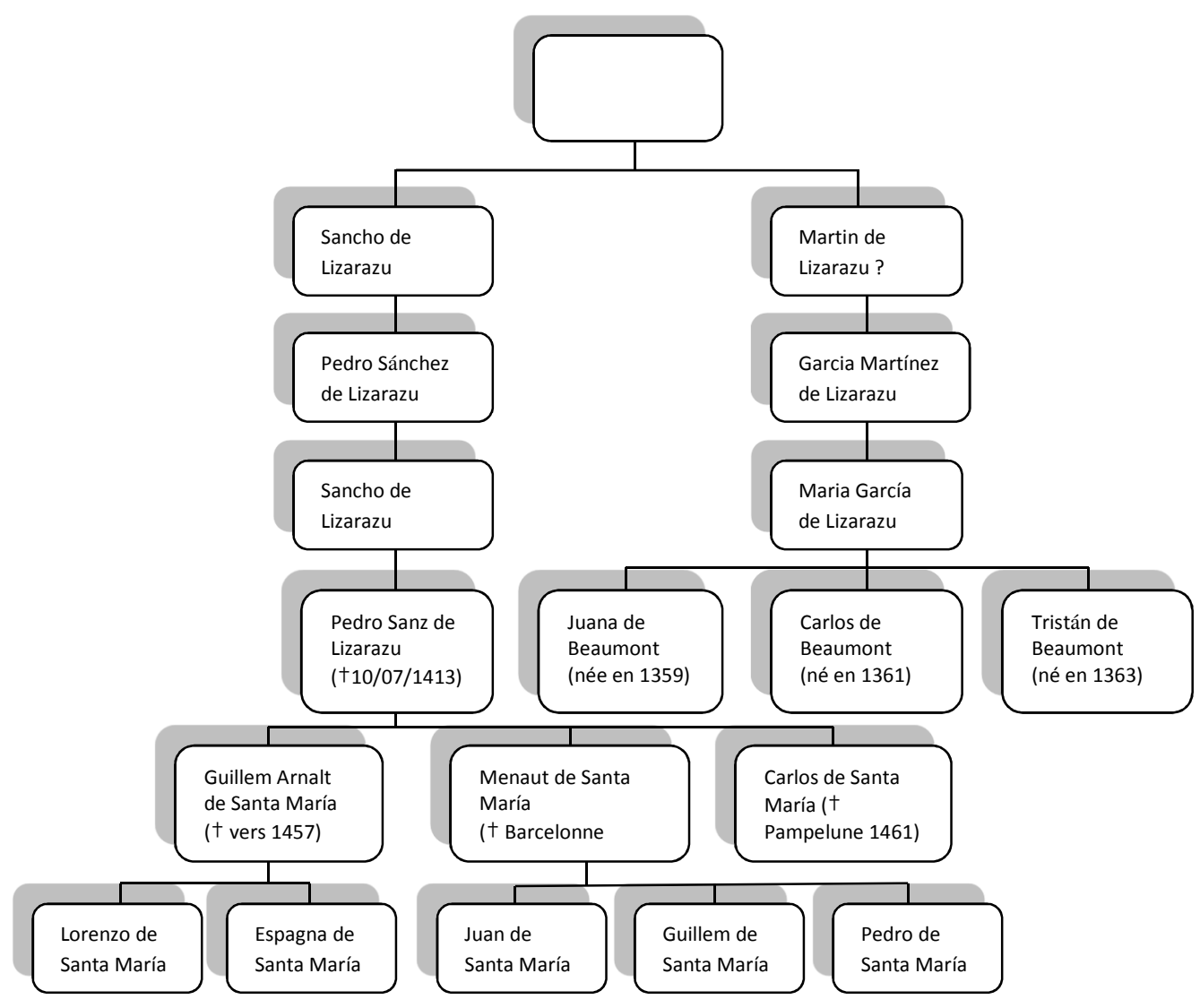

Fig. 1 : la lignée des Lizarazu - Santa María

Le nom Lizarazu, originaire du palais du même nom à Saint-Étienne-de-Baïgorry, se caractérise par une propagation précoce dans l'espace péninsulaire du royaume de Navarre, ce qui conduisit à un contraste intéressant entre la branche principale, qui abandonna ce nom, et les branches secondaires, qui le conservèrent. Fidèles à leur attachement au métier des armes, de plus en plus conscients du rôle du roi en tant que pourvoyeur de grâces et bienfaits, tous les fils de la lignée qui arrivèrent à l'âge adulte exercèrent le métier des armes dans la Navarre du Sud. Nous trouvons de manière permanente une série entière de gouverneurs portant ce nom, surtout dans les forteresses frontalières, très nombreuses du fait de la position géostratégique du territoire (voir carte). 


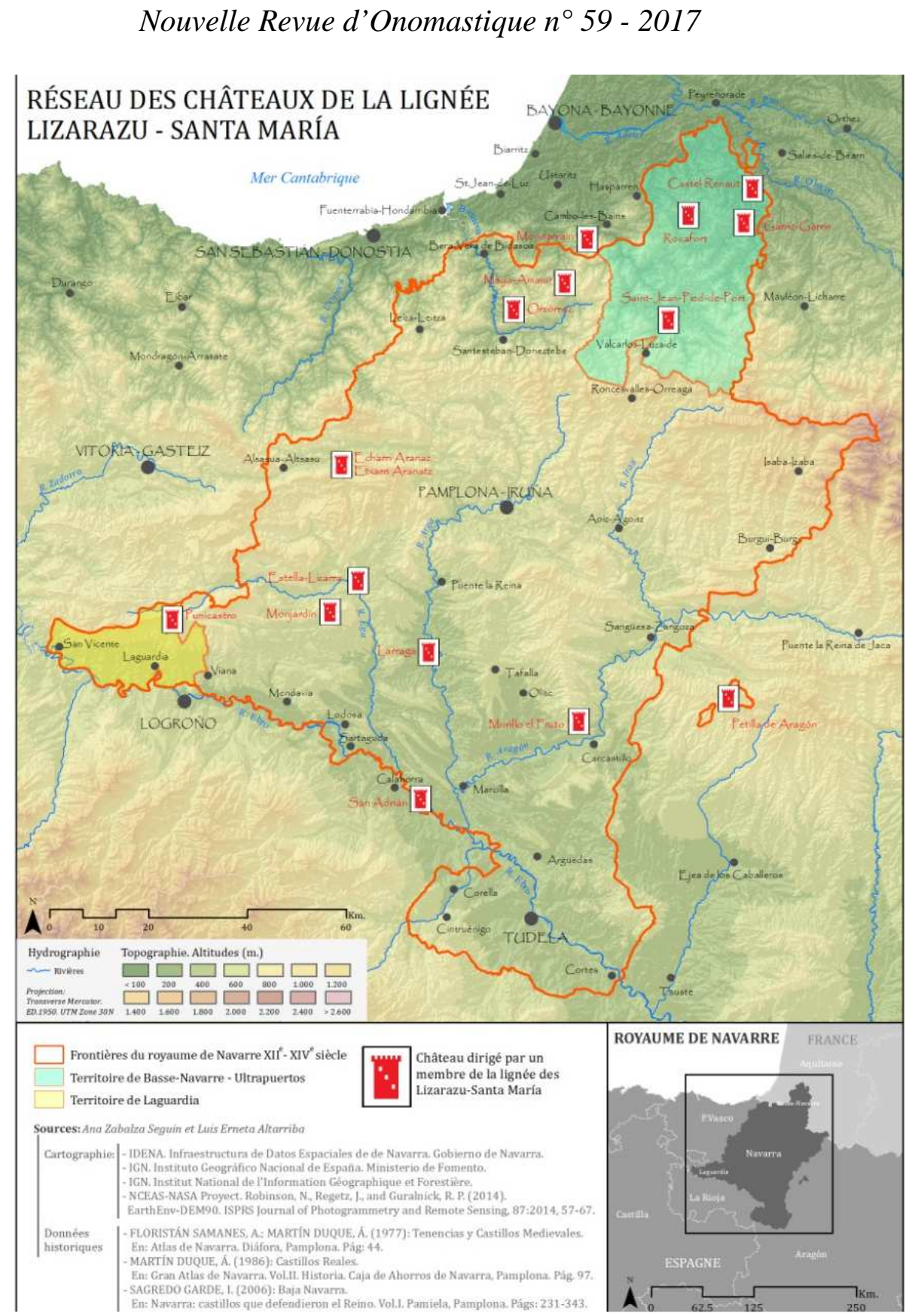

Fig. 2 : les châteaux de la lignée Lizarazu - Santa María

Les Lizarazu sont des hommes de frontières, non seulement parce qu'ils y habitent et vivent grâce à elles, mais aussi parce qu'ils marquent les limites de l'autorité royale. Un 


\section{Au-delà des frontières. Les lignées de Basse-Navarre à la cour du roi}

cas significatif est celui du château de Larraga, véritable belvédère sur la vallée de l'Èbre lors des batailles contre les musulmans et ancien poste frontière, même s'il avait déjà perdu cette fonction. À partir de 1323, on fait état de plusieurs Lizarazu qui se succèdent à la tête de ce gouvernement, en alternance avec d'autres mais de manière permanente. En 1379, Charles II octroie à Juan de Lizarazu les moulins de cette localité à vie, en plus des deux paiements annuels qu'il recevait. Il est significatif qu'en 1390, Charles III accorde au même Juan la somme de 50 florins comme gratification spéciale pour avoir construit des maisons à Larraga, ce qui témoignait de son désir de s'installer dans les terres méridionales avec un lot de biens immobiliers. Malgré tous les événements de la longue guerre civile, les Lizarazu demeurèrent dans cette localité, qui prit une nouvelle valeur lorsque la lignée de Beaumont, prééminente, reçut le comté de Lerín, base territoriale très proche de la ville de Larraga. Ici leur nom se maintint et, selon toute probabilité, il passa de là à la ville voisine de Berbinzana, où il est resté jusqu'au début du $\mathrm{XX}^{\mathrm{e}}$ siècle. Nous pensons que cet enracinement permanent, non d'une branche, mais de plusieurs - Larraga n'est qu'un exemple - confirme l'hypothèse selon laquelle ces lignées avaient le regard tourné vers les terres méridionales, où le service du roi donnait lieu à des récompenses en biens-fonds.

Comme on l'a indiqué plus haut, et c'est compréhensible du fait de la nature des sources, nous connaissons très peu de noms de femmes de cette lignée durant le XIV siècle. Cependant, la seule identifiée avec précision a joué un rôle décisif dans le sort des siens. María García de Lizarazu était la fille de García Martínez de Lizarazu, ce dernier étant sûrement le cousin de Pedro Sanz de Lizarazu, chef de famille de la branche principale au milieu du XIV ${ }^{\mathrm{e}}$ siècle. García était gouverneur du château de Larraga en 1338, et il est très probable que son fils Sancho lui a succédé en 1357. Pendant ces mêmes années, María devint la maîtresse du frère du roi Charles II. L'infant don Luis fut le seul frère du monarque qui survécut à l'âge adulte; à cause des fréquentes absences du monarque du fait de ses campagnes en France, Luis de Beaumont-le-Roger exerça la lieutenance au nom de son frère. Certains auteurs affirment que l'infant Luis et María se marièrent en secret, mais il n'y a aucune preuve documentaire le confirmant. Ce qui est sûr, c'est qu'entre 1359 et 1363, María donna à l'infant trois enfants : Juana (1359), Charles (1361) et Tristan (1363). Il semble que leur relation ait commencé plusieurs années avant la naissance de la première fille, car, à partir de l'été 1356 au moins, María avait reçu des bienfaits qui ne peuvent s'expliquer que par sa relation avec don Luis; si tel est le cas, il se peut qu'elle ait eu d'autres enfants morts très tôt. Le fait est que ces enfants furent les seuls enfants de l'infant, car, marié par la suite avec Juana de Durazzo (1366) - mariage purement politique -, il n'eut pas de descendance légitime. Les trois enfants de María de Lizarazu seront connus sous le nom de Beaumont, emprunté au comté dont son père était titulaire. Du second enfant, Charles de Beaumont, naîtra la lignée de ce nom, qui prendra la tête du camp du même nom pendant la guerre civile. Depuis le milieu du XIV ${ }^{\mathrm{e}}$ siècle, les Lizarazu ont donc fait partie de manière illégitime de la famille royale, un fait qui décidera de leur destin. À tout moment, ils feront en sorte de maintenir fermement leur position dans leur pays natal - une tâche qui incombe à l'aîné de chaque branche -, mais sans abandonner la présence à la Cour, un nœud où se mêlent tous les fils qui composent le creuset du royaume de Navarre, 
lieu où l'information non seulement se transmet mais aussi se crée ; et tout cela sans abandonner leur position à la tête de différents châteaux.

Le moment où se noua la discrète parenté des Lizarazu avec la dynastie royale est significatif. La relation de don Luis et María dut commencer vers 1355-1356, c'est-à-dire sept ans à peine après la catastrophe de la peste noire. Ses conséquences furent dramatiques pour la Navarre, et c'est peut-être dans ce contexte qu'il faut comprendre l'importance des relations hors mariage et de la descendance illégitime, phénomène qui affecte les membres des familles royales et de la noblesse. Il est facile de comprendre que devant des circonstances que l'homme ne peut prévoir ni combattre, le désir de laisser une descendance se soit accru. De ce point de vue, les titres des Lizarazu étaient excellents, car, comme on peut le constater en reconstruisant leurs biographies, ils se distinguent par leur force physique, leur longévité et leur descendance prolifique, au moins en ce qui concerne les hommes. D'ailleurs, certains étaient connus par des surnoms tels qu'Héruy, Sanson ou Gaillard. Est-ce cela que recherchait don Luis, qui serait empêché de se marier avec María à cause de la lignée inférieure des Lizarazu ?

En ce qui concerne l'onomastique des frères Beaumont, il est intéressant de constater une certaine oscillation, car, si pour le premier fils de chaque sexe on choisit des noms patrimoniaux de la dynastie royale - Juana était le nom de la reine, mère de don Luis, et Charles, celui de son frère le roi, son parrain sûrement -, en revanche pour le plus jeune, on préfère un nom moins traditionnel, peut-être emprunté à la littérature : Tristan. Cette pratique sera adoptée par la famille royale navarraise au moment de baptiser sa nombreuse descendance illégitime avec des prénoms comme Lionel, Lancelot ou Godefroy, tandis que, pour la descendance légitime, elle utilisera plutôt un répertoire plus traditionnel.

Passons à présent à l'analyse des noms et prénoms de la branche principale de la lignée. Il faut tenir compte du fait que les sources que nous avons utilisées sont rédigées en langue romane, beaucoup d'entre d'elles en langue romane navarraise, qui finit par disparaître ou s'assimiler au castillan. Cependant, on peut supposer que la langue maternelle des Lizarazu était la langue basque, qui ne s'utilise dans la documentation que de manière exceptionnelle. Donc, à cet archaïsme que l'on peut supposer dans les textes officiels, il faut ajouter la difficulté qu'implique la transcription des sons qui n'ont pas de correspondant exact en langue romane, comme c'est le cas pour les lettres $c ̧$ ou $z$ dont on trouve deux occurrences dans le nom de la lignée. Jusqu'à la conquête castillane du royaume de Navarre (1512), voire jusqu'à l'abandon par la Castille des territoires de BasseNavarre (vers 1530), les gens originaires de ces terres passaient librement d'un côté et de l'autre des Pyrénées. À partir de 1583, par décision des Cortes (le Parlement) de Navarre, ils furent privés de leur identité de Navarrais et tenus pour des étrangers en ce qui concerne les métiers et les bénéfices. Pour cette recherche, nous avons utilisé des documents émis par un organisme central du royaume, la Cámara de Comptos; nous centrons donc l'analyse sur la façon dont ces noms ont été transcrits au sud des Pyrénées, lieu où une bonne partie de la lignée s'est finalement ancrée.

Les quatre premières générations de la branche principale, entre 1338 et 1413 , suivent rigoureusement le schéma de transmission du nom du grand-père paternel au petit- 


\section{Au-delà des frontières. Les lignées de Basse-Navarre à la cour du roi}

fils héritier : Sancho - Pedro - Sancho - Pedro. Tous hommes d'armes, ils assurèrent la charge de gouverneur de différents châteaux; la source la plus ancienne présente Sancho de Lizarazu comme gouverneur du château de Larraga en $1323^{5}$. Ce document le présente déjà comme un homme d'armes au service du roi partout où cela est nécessaire - en l'occurrence, loin de sa terre natale -, mais, de plus, il a manifesté, par le choix de son épouse, sa volonté de s'installer dans la Navarre péninsulaire : en effet, il se marie en secondes noces avec l'héritière d'Ursúa, à Baztán 6 , une lignée frontalière avec laquelle ils devaient établir un nouveau lien quatre générations plus tard. Ce premier Sancho et ses contemporains durent être touchés par la grande épidémie de peste de 1348 ; peut-être cela explique-t-il la mort de la première épouse, dont nous ignorons le nom, et le second mariage entre deux héritiers, celui de Lizarazu et celle d'Ursúa, une situation qui ne se produit généralement qu'en temps de crise grave, car elle porte atteinte au principe fondamental qui est en usage partout où les biens se transmettent à un seul héritier, à savoir que le même nombre d'unités domestiques doit se maintenir à chaque génération ${ }^{7}$. Quoi qu'il en soit, Sancho eut au moins deux fils et put transmettre à chacun un patrimoine complet : à Pedro, issu peut-être du premier mariage, celui de Lizarazu, et à Miguel Sanz, fils du second mariage, celui d'Ursúa, la tradition étant ainsi rétablie pour la génération suivante. Ce lien de sang étroit peut contribuer à expliquer pourquoi les Lizarazu laissèrent passer presque un siècle avant de revenir chercher un conjoint chez les Ursúa. Íñigo Sanz de Lizarazu, gouverneur de Punicastro en $1350^{8}$, et García Sanz ou Sánchez de Lizarazu, qui se trouvait à la tête du château pyrénéen de Mondarráin en 1351 et en $1363^{9}$, pourraient aussi être des fils de Sancho ${ }^{\text {er }}$. D'autres documents nous le présentent comme homme d'armes au service du roi : ainsi, pendant l'été 1353, il fut l'un des émissaires de Charles II en Normandie ; avec Íñigo Sánchez de Ursúa, il apporta vingt-huit hommes de pied ${ }^{10}$. En août 1362, García toucha une certaine somme du trésorier du royaume pour les dépenses des gens d'armes pendant la guerre d'Aragon ${ }^{11}$. Aussi bien Íñigo que García portaient le nom d'anciens rois autochtones. Chacun de ces hommes a donné naissance à de nouvelles branches de la lignée, d'où ont continué à surgir des soldats, mais aussi des officiers de l'administration du royaume. Il en fut ainsi avec les enfants d'Íñigo Sánchez de Lizarazu, cité plus haut : Sancho Î́niguez de Lizarazu, qui reçoit le nom de son grand-père, est le seigneur du palais d'Echaide, à Elizondo, et gardien du château d'Orzórroz, à Baztán, en

\footnotetext{
${ }^{5}$ MARTinena Ruiz 1994, p. 639.

${ }^{6}$ RAMÍREZ VAQUERO 1990, p. 114.

${ }^{7}$ Moreno Almárcegui et al. 1999, p. 274-277.

${ }^{8}$ Martinena Ruiz 1994, p. 659. Dans les documents de la Cámara de Comptos, on trouve des informations sporadiques le concernant, à la tête de ce château, entre 1351 et 1357 : Archivo General de Navarra [AGN], Co_documentos, caj. 11, n. 22, 1.

${ }^{9}$ AGN, Co_documentos, caj. 11, n. 28 et caj. 17, n. 40 (1) ; MARTinena RuIZ 1994, p. 647.

${ }^{10}$ FERNÁNDEZ DE LARREA RoJas 1992, p. 99.

${ }^{11}$ AGN, Co_documentos, caj. 15, n. 93, 26.
} 
1351, tandis que son frère, Juan Íñiguez de Lizarazu, fut notaire de la cour, même s'il participa aussi aux campagnes de Normandie ${ }^{12}$.

Les ramifications de l'arbre généalogique sont remarquables, mais nous devons revenir à la branche principale. Le fils aîné de Sancho I ${ }^{\text {er }}$, Pedro Sánchez de Lizarazu, attesté entre 1347 et 1363, fut écuyer et sergent d'armes ; il participa également à la campagne de Normandie pendant l'automne 1353 avec ses hommes, recevant sa paye de la part du roi ${ }^{13}$. Il est contemporain de sa cousine María, et sa proximité avec le monarque se perçoit par exemple dans le fait qu'avec son fils héritier Sancho, il accompagne le roi lors de son voyage en France ${ }^{14}$. Ses fonctions sont variées, car il apparaît tantôt comme gouverneur de châteaux (Mondarráin, Murillo, San Adrián), tantôt exerçant la charge de baile, c'est-à-dire en tant que représentant du roi à Labastide-Clairence. Nous ignorons le nom de son épouse.

On retrouve son fils Sancho dans des documents de 1353 et 1378, bien que cette information soit insuffisante. En revanche, il semble qu'il fut l'homme de confiance de Charles II : en effet, en 1362, le monarque ordonne qu'on lui remette dix florins pour une cause secrète ${ }^{15}$.

\section{Pedro Sanz de Lizarazu, chef de la lignée (1375-1413)}

Le fils de Sancho, Pedro Sanz de Lizarazu, est certainement la figure la plus remarquable de toute la lignée, c'est du moins ce que l'on déduit de la documentation qui est parvenue jusqu'à nous. Cité dans les documents dès 1375, il est quasiment certain qu'il mourut le 10 juillet 1413. Il vécut donc sous le règne de Charles II et de Charles III, sans que sa position ait été affectée par le passage de l'un à l'autre. Étant, comme nous le verrons, un personnage important à la Cour, il se situait dans une position de subordination par rapport aux Beaumont, les descendants illégitimes du côté masculin de la famille royale elle-même et d'une Lizarazu. Au fur et à mesure que s'écoulait le règne de Charles III et que les grandes lignées prenaient l'habitude de se partager les rentes du royaume, la division entre les descendants illégitimes de Charles II, les Peralta-Agramont, et ceux de l'infant Luis, les Beaumont, parmi lesquels étaient les Lizarazu, commença à se manifester. Mais, avant le déclenchement du conflit dynastique en 1450-1451, il s'agissait d'une situation analogue à celle qui caractérisait les cours des royaumes voisins.

Dans la biographie de Pedro Sanz, on peut distinguer plusieurs aspects. Comme les autres hommes de sa lignée, il fut avant tout un homme d'armes, qui exerça toute sa vie le gouvernement de différents châteaux qui lui furent confiés, en particulier non loin de sa terre natale, en Basse-Navarre, ceux de Castelrenaut (1388), et, pendant longtemps, celui de Rocafort (1388-1401). Alors que d'autres engagements l'empêchaient d'y séjourner, il

${ }^{12}$ AGN, Co_documentos, caj. 12, n. 98 ; FERNÁNDEZ DE LARREA RoJAs 1992, p. 120.

${ }^{13}$ AGN, Co_documentos, caj. 12, n. 23, 26.

${ }^{14}$ AGN, Co_documentos, caj. 12, n. 22, 4.

${ }^{15}$ AGN, Co_documentos, caj. 15, n. $88,7$. 


\section{Au-delà des frontières. Les lignées de Basse-Navarre à la cour du roi}

confia cette dernière charge à un lieutenant. Uniquement à la fin de sa vie, peut-être en guise de récompense, il reçut la châtellenie de Saint-Jean-Pied-de-Port, la plus importante de Basse-Navarre, ainsi que celle du château de Garris sur ce même territoire ; il exerça ces deux fonctions jusqu'à sa mort ${ }^{16}$.

Par ailleurs, Lizarazu figure depuis 1376 en tant que bénéficiaire des terres de Mixa et Ostabarets. Après la mort de Charles II le premier jour de 1387, il reçut bientôt de nouvelles récompenses de son successeur ; ainsi, dès 1390, il perçut chaque année le tribut de la vallée d'Arce comme don à vie pour maintenir sa troupe de gens d'armes ; on trouve mention de ce tribut jusqu'en 1411, quelques mois avant sa mort ${ }^{17}$. D' autre part, Charles III le fit chevalier l'année de son couronnement, en $1390^{18}$.

Ce qui attire l'attention chez ce personnage, c'est sa capacité à se maintenir actif sur différents fronts de manière simultanée ; ainsi, fin 1399, il apparaît comme grand maître de l'hôtel du roi, métier dans lequel se distinguera plus tard un de ses enfants. Une charge que ses descendants et lui effectueront à plusieurs reprises est celle d'ambassadeurs et de messagers du roi. Pedro avait déjà commencé cette délicate mission sous le règne de Charles II, lorsque, par exemple, on l'envoyait à Lourdes : Lizarazu était parfaitement habilité pour agir comme lien entre la cour et la Basse-Navarre; c'était sa fonction naturelle. Sa relation étroite avec les Beaumont fit qu'il assura aussi le lien avec l'Angleterre, car cette lignée favorisait l'influence des Anglais sur le territoire français. En 1397, il accompagna le roi lors de son voyage en France. Déjà sous le règne de Charles III, il avait été envoyé en Béarn (1392), en Aragon (1393), en Angleterre (1396), à Foix (1399), en Béarn à nouveau (1406), en Castille (1406), en Basse-Navarre et à Bayonne (1407). Signe de sa proximité avec le monarque et de sa position à la Cour, lorsque Charles III revint en Navarre après un long voyage en France, en septembre 1398, il s'arrêta pour manger à l'hôtel de Pedro Sanz de Lizarazu ${ }^{19}$.

À six reprises au moins, Pedro Sanz reçut du monarque un présent très spécial : un cheval, instrument indispensable pour ses missions au service de la Couronne. Il faut souligner ici le grand laps de temps écoulé entre le premier et le dernier cheval offert : il s'est en effet passé trente ans entre le cheval acheté sur l'ordre de Charles II en $1381^{20}$ et le dernier, offert par Charles III en juin $1411^{21}$. Il faut l'interpréter comme un signe de la force physique qui semble caractériser les Lizarazu. Sa mort, à l'été 1413, coïncida avec une réunion du Parlement à Olite ; quelques jours plus tard mourrait aussi l'infante aînée, Juana, et deux autres grands personnages de la Cour. Tout cela fit que leurs funérailles eurent lieu dans l'église du couvent San Francisco de cette localité pendant quatre jours consécutifs du mois de juillet ${ }^{22}$. Cela indique bien sa position à la Cour.

\footnotetext{
${ }^{16}$ AGN, Co_documentos, caj. 106, n. 3, 31.

${ }^{17}$ AGN, Co documentos, caj. 59, n. 87, 1.

${ }^{18}$ CASTRO 1967 , p. 421.

${ }^{19}$ CASTRO 1967, p. 243.

${ }^{20}$ AGN, Co_documentos, caj. 43, n. 39, 1.

${ }^{21}$ AGN, Co_documentos, caj. 98, n. 61, 2 (1).

${ }^{22}$ CASTRO 1967, p. 392.
} 
Du point de vue de l'onomastique, Pedro Sanz montre un changement notable dans la trajectoire de la lignée. Pour ses quatre fils attestés dans la documentation - il faut supposer qu'ils naquirent autour de la décennie 1380 -, il choisit des noms qui s'écartent de la tradition familiale. En effet, la liberté avec laquelle on procéda dans le cas du fils aîné, pour lequel on aurait pu s'attendre à une plus grande fidélité à la norme, attire notre attention : celui-ci s'appelle Guillem Arnalt, nom très fréquent en Basse-Navarre, mais complètement étranger à la tradition familiale. Comme on l'a déjà signalé, on peut toujours supposer qu'il eut un premier fils appelé Sancho - comme la tradition l'exigeait - qui serait mort très tôt sans laisser aucune trace ; malgré tout, il existait la coutume du remplacement, c'est-à-dire qu' un autre frère, né après lui, recevait son nom ; s'il n'y avait pas de nouvelles naissances, on pouvait aussi changer le nom d'un frère. Cependant, rien de tout cela n'est attesté pour les enfants de Pedro Sanz. Le fait qu'au moins trois fils aient suivi l'aîné contribue à réaffirmer l'hypothèse selon laquelle il s'agit d'une décision préméditée ; un autre des frères, le plus documenté, porte tout comme l'aîné un nom de tradition germanique, relativement commun en Basse-Navarre, Menaut; un autre s'appelle Charles, fait absolument exceptionnel dans ce pays - comme le montre Orpustan dans ses études détaillées $^{23}$ - et qui ne peut s'expliquer que parce qu'il serait le filleul du monarque ; le moins connu est Juan, qui, fait particulier, apparaît dans les sources sous le nom de Juan Pérez, c'est-à-dire avec son patronyme, ce que l'on ne retrouve pas dans le cas de ses frères. Malgré la riche documentation où apparaissent ces personnages, je n'ai pu déterminer le nom de l'épouse de Pedro Sanz, ni si ces enfants sont issus ou non du même mariage.

Le renouvellement onomastique opéré dans cette lignée à la fin du XIV e siècle est un fait. Comment l'interpréter ? Pedro Sanz de Lizarazu a pu pressentir que les grâces qu'il espérait obtenir ne lui seraient pas concédées par un roi nommé Pedro ou Sancho, mais par Charles. Il se peut aussi qu'il ait recherché pour parrains de ses enfants des personnages de la cour - où les Bas-Navarrais étaient nombreux - qui portaient effectivement les noms cités. La question reste ouverte, et il est difficile d'éviter la conjecture : en fin de compte, à l'attribution réfléchie d'un prénom à un nouveau-né vient s'ajouter une certaine dose de hasard, de caprice ou de mode, qui résulte de circonstances de dernière minute. Mais ce qui est réellement important, c'est qu'en plus de la nouveauté du prénom, il y eut changement de patronyme : tous les enfants de Pedro, y compris l'héritier, abandonnèrent celui de Lizarazu pour celui de Santa María, nom d'un autre de leurs palais, situé dans le village d'Hélette (Arberoue). Tout le monde sait qu'au Moyen Âge, voire à l'époque moderne, régnait une grande liberté dans l'utilisation des noms ; ce que, de notre perspective, nous considérons comme un nom était en fait une référence au domaine, à la terre. De ce point de vue, les Lizarazu énumèrent fièrement plusieurs terres : Lizarazu, Gentain, Egoaburua, Santa María. Même Pedro fut parfois appelé Pedro Sanz de Santa María. Mais, à cause précisément de la flexibilité qui prévalait en ce domaine, le fait que le nom de Lizarazu cesse brusquement d'être porté - il ne s'appliquera que très rarement à l'un des quatre frères - ne peut manquer d'attirer l'attention. On peut supposer qu'il y eut discussion au moment

${ }^{23}$ ORPUSTAN 2000. 


\section{Au-delà des frontières. Les lignées de Basse-Navarre à la cour du roi}

d'écarter l'un des noms de référence au profit d'un autre. Avec la rupture de la tradition dans les prénoms, la conclusion que l'on peut tirer est que Pedro Sanz de Lizarazu chercha consciemment à imprimer un changement d'orientation à sa lignée. Cette hypothèse est confirmée par la politique matrimoniale suivie pour la génération de ses enfants, qui lui est sans aucun doute imputable, et que nous connaissons seulement de manière fragmentaire : cette génération sera victime de la guerre civile, pendant laquelle elle se retrouvera au cœur même de la lutte, mais les deux frères que nous connaissons le mieux, Guillem Arnalt et Menaut, se marièrent avec des dames de la péninsule. Le premier épousa Margarita de Uroz-Ursúa, renouant ainsi avec une importante maison, avec laquelle les Lizarazu avaient déjà eu des liens de parenté autrefois ; le second, Catalina de Aoiz, née dans un palais dont les enfants servaient à la cour d'Olite, comme les Lizarazu.

Avant de passer à l'interprétation du sens possible de cet apparent changement de cap, il convient d'examiner si, pour leur part, les enfants de Pedro Sanz de Lizarazu suivirent la tradition ou continuèrent à innover. Comme je viens de le signaler, le long conflit qui dévasta la Navarre à partir de 1451 provoqua une importante altération de la production documentaire ; la Cour en tant que telle cessa de fonctionner normalement, des pertes et des pillages affectèrent la conservation des documents. Malgré tout, parmi la descendance relativement nombreuse de Guillem Arnalt et de Menaut, on observe un certain mélange de nouveauté et de tradition - même si prédomine peut-être la nouveauté. Menaut donne à un de ses enfants le nom de son père, Pedro, et aux deux autres ceux de ses frères, Guillem et Juan. En revanche, le fils aîné semble opter pour des noms nouveaux ou peut-être empruntés au patrimoine des Ursúa, la lignée de son épouse, tels que Beltrán et Lorenzo. Contrairement à ce qui se passait dans les générations précédentes, les femmes commencent alors à émerger, bien qu'en nombre très inférieur à celui des hommes; de plus, comme nous manquons cruellement de données antérieures, il est impossible d'analyser les critères de transmission. Une des filles de Menaut fut nommée Ana; mais le fait le plus significatif est peut-être que le fils aîné Guillem Arnalt ait baptisé la sienne du nom d'España. La petite-fille du côté masculin de la primogéniture de Pedro Sanz de Lizarazu portait le nom d'España de Santa María.

Dans son étude consacrée à l'anthroponymie de la Basse-Navarre et de la Soule dans la première moitié du XIV ${ }^{\mathrm{e}}$ siècle - soit un siècle avant la naissance d'España de Santa María -, ORPUSTAN identifie comme trait caractéristique de l'anthroponymie féminine malgré le nombre insuffisant d'exemples, 95, face aux 911 hommes - la proportion élevée de noms qu'il qualifie d' «ethniques »: Navarra, Alamana, Espaynna, Anglesa, Lombarda. Cet auteur considère qu'il s'agit de noms à la mode et les met en relation avec les routes suivies par les armées ou par les pèlerins ${ }^{24}$. Selon lui, et c'est une interprétation intéressante, cette mode reflèterait une certaine ouverture culturelle vers les peuples d'Europe cités : Hispania, dans le cas de l'Espagne, entité disparue qui englobait les territoires de la Péninsule Ibérique, mais dont l'essentiel demeurait compris dans les royaumes chrétiens. En tout cas, dès la seconde moitié $d u \mathrm{XV}^{\mathrm{e}}$ siècle, époque à laquelle vivait la fille de Guillem

${ }^{24}$ Orpustan 2000, p. 201-202. 
Arnalt, ce nom passait pour archaïque : s'il avait pu être à la mode, il ne l'était déjà plus à ce moment-là. On peut peut-être dégager de tout cela une déclaration d'intentions : la lignée, déjà du vivant de Pedro Sanz de Lizarazu, cherche à s'hispaniser. C'est peut-être dans ce désir que se trouve l'explication du brusque abandon du nom, un mot basque difficile à prononcer et à écrire, qui renvoyait, ne serait-ce que par sa sonorité, au monde du saltus vasconum, au milieu rural, loin de la culture et de l'écrit. La vie de Pedro Sanz s'était déroulée entre les deux mondes, mais pesa sans doute chez lui de manière décisive celui de la Cour alors à son apogée : comme on l'a récemment écrit, dans toute l'histoire du royaume de Navarre, il n'y eut pas de mécène plus engagé dans la défense des arts que Charles III, et la cour du roi ne brilla sûrement jamais autant ${ }^{25}$. Les enfants de Pedro, en particulier Menaut - majordome du prince de Viana, don Carlos, depuis son arrivée en Navarre à l'âge d'un an, en 1422, jusqu'à sa mort en 1460 ou 1461 -, vécurent et servirent à la Cour. Menaut de Santa María, avec ses enfants et ses neveux, accompagna le prince héritier pendant tout son long périple jusqu'à Barcelone ; là, malgré sa situation difficile, don Carlos, en homme de culture, prit grand soin de sa bibliothèque, à la tête de laquelle il plaça un remarquable écrivain en langue catalane, frère Pere Martínez, qu'il chargea d'un inventaire que Menaut lui-même signa très peu de temps avant sa mort ${ }^{26}$. Le nom de ses enfants, décidé, comme pour toute sa trajectoire, par Pedro, avait eu pour objectif de faciliter son insertion dans cette sphère supérieure : le nom rustique de sa terre natale représentait plus un obstacle qu'une aide.

\section{Conclusion}

Si l'intuition de Pedro, dès les années 1380-1390, était qu'il fallait parier sur les territoires péninsulaires, il eut certainement raison. Ce qu'il n'avait pu prévoir, c'est que la fracture entre les deux grandes factions s'aggrava lorsqu'elle s'ajouta au conflit dynastique qui suivit la mort de la reine Blanca, fille de Charles III (1441). À partir de ce moment, les enfants et petits-enfants de Pedro furent entraînés dans un affrontement long et coûteux, dans lequel ils jouèrent un rôle important, bien que subordonnés aux Beaumont, chefs de file d'une des factions. En faisant respecter les droits du prince héritier, Carlos de Viana, face à ceux de son père, Juan II de Aragón - qui, veuf de la reine en titre, remarié en secondes noces avec une castillane, prétendait continuer à être roi de Navarre - ils allèrent jusqu' au bout et payèrent un prix élevé. Guillem Arnalt fut dépossédé de son château ; un de ses enfants fut échangé contre la liberté du prince ; Menaut vit brûler son palais d'Urroz; avec ses enfants, il suivit le prince jusqu'à Barcelone, où il mourut presque en même temps que don Carlos, en 1460 ou 1461, dans des circonstances pour le moins suspectes. Quant au troisième frère, Carlos, aux alentours de septembre 1461, alors que la cause du prince de Viana était perdue, il fut condamné pour rébellion et désobéissance au roi, puis exécuté,

\footnotetext{
${ }^{25}$ FERNÁNDEZ-LADREDA 2015, p. 18.

${ }^{26}$ LATASSA Y ORTín 1796, t. II, p. 229-230.
} 
Au-delà des frontières. Les lignées de Basse-Navarre à la cour du roi

et tous ses biens confisqués et livrés à ses ennemis ${ }^{27}$. Cette série de malheurs ne signifia pas la fin des Lizarazu-Santa María. Au moins deux des enfants de Menaut, Guillem et Juan, avaient accompagné leur père à Barcelone, où Juan épousa une dame catalane, Violant Satorra; son frère apparaît dans la documentation barcelonaise comme le molt honorable mossen Guillem de Sancta Maria. En quelque sorte, Pedro Sanz de Lizarazu avait concentré tout son effort pour préparer les siens à s'hispaniser, et c'est finalement ce qui arriva, même si ce fut par des voies très différentes de celles qu'il avait imaginées.

\section{Bibliographie}

Atlas de Navarra : geográfico, económico, histórico, 1977, Barcelone, Diáfora.

CASTRO José Ramón, 1967, Carlos III el Noble, Rey de Navarra, Pampelune, Institución Príncipe de Viana.

FERNÁNDEZ DE LARREA RoJAS Jon Andoni, 1992, Guerra y sociedad en Navarra durante la Edad Media, Bilbao, Universidad del País Vasco.

FERNÁNDEZ-LADREDA Clara (dir.), 2015, El arte gótico en Navarra, Pampelune, Gobierno de Navarra.

Floristán SAMANES Alfredo et MARTín DuQue Ángel J., 1986, Gran atlas de Navarra, Pampelune, Caja de Ahorros de Navarra.

Fortún PÉREZ DE CIRIZA Luis J. et FloRISTÁn IMÍZCOZ Alfredo, 2008, Navarra, Los límites del Reyno, Pampelune, Gobierno de Navarra.

LATASSA Y ORTíN Félix, 1799, Biblioteca nueva de los escritores aragoneses, t. II, Pampelune, Joaquín de Domingo.

MARTINENA RUIZ Juan José, 1994, Castillos reales de Navarra (siglos XIII al XVI), Pampelune, Gobierno de Navarra.

— 1997, Guía del Archivo General de Navarra, Pampelune, Gobierno de Navarra.

Moreno Almárcegui Antonio et Zabalza SEguin Ana, 1999, Origen histórico de un sistema de heredero único : el Pre-Pirineo navarro, 1540-1739, Madrid, Rialp.

ORPUSTAN Jean-Baptiste, 2000, «Anthroponomastique médiévale en Pays basque: prénoms et surnoms en Basse-Navarre et Soule au debut du XIV ${ }^{\mathrm{e}}$ siècle (13051350) », Lapurdum, $\mathrm{n}^{\circ} \mathrm{V}$, p. 183-221.

Procesos de hidalguía del Consejo Real de Navarra que se conservan en el Archivo Real y General de Navarra : siglo XVI, 2015, Madrid, Hidalguía.

RAMÍREZ VAQUERO Eloísa, 1990, Solidaridades nobiliarias y conflictos políticos en Navarra, 1387-1464, Pampelune, Gobierno de Navarra.

SAGREDO Iñaki, 2006, Navarra : castillos que defendieron el Reino, Pampelune, Pamiela. WRIGLEY Edward Anthony (éd.), 1973, Identifying people in the past, Londres, Arnold.

\footnotetext{
${ }^{27}$ Procesos de hidalguía 2015, p. 55-56.
} 
Nouvelle Revue d'Onomastique $n^{\circ} 59$ - 2017

ZONABEND Françoise, 1979, «Jeux de noms : Les noms de personne à Minot », Études rurales, $\mathrm{n}^{\circ} 74$, p. $51-85$. 